\title{
SIOP/COG/NWTSG Stage III
}

National Cancer Institute

\section{Source}

National Cancer Institute. SIOP/COG/NWTSG Stage III. NCI Thesaurus. Code C140305.

Residual Wilms' tumor confined to the abdomen; Lymph nodes in the renal hilum, the periaortic chains, or beyond are found to contain tumor; diffuse peritoneal contamination by the tumor; implants are found on the peritoneal surfaces; tumor extends beyond the surgical margins either microscopically or grossly; tumor is not completely resectable because of local infiltration into vital structures. 